\title{
Emotion Communications Driven by Big Data Cloud, Smart Clothing and Robotics
}

\author{
Jeungeun Song, Mengchen Liu, Gaoxiang Wu, Lu Wang, Yunhao Li, \\ Enmin Song* \\ *Corresponding author: Enmin Song \\ School of Computer Science and Technology, Huazhong University of Science and \\ Technology, Wuhan 430074, China \\ j song@hust.edu.cn, mengchenliu.cs@qq. com, gaoxiangwu. epic@qq. com, luwang. \\ epic@qq.com, liyh.epic@qq.com, esong@hust.edu.cn
}

\begin{abstract}
The functions of the multi-networked machines far exceed those of independent and isolated ones. The machines will upload the human-related signals and environmental signals that they have collected to the cloud through the Internet, then the cloud will offer powerful intelligent processing which will process the data and send it back to another machine (i.e. the robot), and the robot will be used to serve the people, thus a circuit will be formed. If the robot is able to understand peoples emotions with the help of the emotional big data analysis, it will make it possible for the emotion communication.

Briefly, our core concept is to start from people to Smart Clothing, then from Smart Clothing to the cloud, next from the cloud to the robot, and at last from the robot to people again. So the important elements of this system include Smart Clothing, Health Big Data Cloud and robots.

The Internet of Things interconnects things to things, which realizes the communication in the physical world, and then this paper mainly discusses the emotion communication problems in the spiritual world. In order to solve this challenging problem, first, well take the application of the big data in the healthcare field as an example, and put forward four strategies and steps to solve the problem through the big data. Then were going to introduce Smart Clothing, a comfortable and intelligent wearable device used to collect the raw data that is the closest to the human bodies. Finally, we will introduce the emotion-care robots.
\end{abstract}

\section{The Internet of Things: Communications among Things}

Our world is interconnected with countless things through the Internet and mobile phones, so the demand for the seamless integration between the physical world and the information world is becoming higher and higher. Meanwhile, we can predict that our human society as well as the interconnection between the physical world and the spiritual world will become the development trend of the future network [1]. When we talk about the emotion communication, we first need to understand how the connected machines communicate with each other $[2,3,4]$. The communication and interconnection among plenty of connected 
machines are also called the Internet of Things, which includes a lot of technology such as wireless sensor network, body area network, pervasive computing, M2M, cloud computing, etc $[5,6,7]$. However, the Internet of Things isn't a static frame or concept; instead, it has evolved into various kinds of advanced technology, such as 5G Network and the big data [8, 9].

Many people divide the architecture of the Internet of Things into four layers, namely the sensing layer, network layer, analysis layer and application layer. We think that there are different interactions among these layers. For example, the interaction between the analysis layer and the sensing layer can realize the energy consumption optimization of the sensor network based on the cloud integration, that is to say, the wisdom gained from the cloud will make the wireless sensor network more intelligent. Sometimes, if the users interact with the lower layers, they will get the user-centered data analysis, personalized sensing Internet of Things, etc [10].

Generally, the Internet of Things includes four logic chains: first, we need different sensors, equipments and machines to perceive the data and the data in the sensors will be collected to the cloud through various kinds of wireless networks and heterogeneous network technology, and finally the data will be analyzed in the cloud [11, 12]. Through the big data analysis, we can learn knowledge and find optimized solutions to change the environment or predict the things that will happen in the end. The whole procedure involves machines, people, cloud computing, big data, and various kinds of learning and analysis technology.

Based on the framework of the Internet of Things, we are able to dig into the deeper-level problems: how can we design plenty of powerful sensors on single equipment, how can we monitor any system anytime anywhere and how can we find out valuable information and make predictions in real time; in order to realize this dream, we need to have the cloud, big data, cloud analysis, machine learning, inference and control.

\section{Emotion Communications in the Spiritual World}

Emotion communication means that a system is able to collect humans emotion data, understand humans emotions, care for peoples feelings and have an effective interaction with the users. Emotion care is a specific field in the healthcare domain, which faces a lot of challenging problems. The emotion communication system put forward in this paper involves medical big data, Smart Clothing and emotion care robot technology. The Smart Clothing constitutes the front-end raw material collection, and upload the data to the data center through mobile phones for processing, and then the robots will realize the action feedbacks. 


\section{Medical Big Data}

We think that the biggest problem of the big data is the source of the data, with these data coming from the source rather than processed one as much as possible, so we need to work with the data owners. Then we need to discuss with the domain experts to find out the unresolved problems, and finally, we have to set up a mathematical model, design algorithm, and program implementation about this problem, thus a data science team is formed. For example, for the medical big data, we need to discuss with the doctors.

Since we are concentrated on the healthcare field, we take the healthcare as an example and summarize four strategies, with them being summarizing problems (scientific problems and those haven't been solved), collecting data, selecting technology and methods, and making correct decisions respectively. Were going to talk about these four problems respectively below.

\subsection{Correct Problems}

First, we need to think about what kind of problems we need to solve. The healthcare field is the biggest problem in the USA, with $20 \%$ of American GDP being invested to solve the medical problems. $50 \%$ of Americans have one or more chronic diseases in varying degrees, and $80 \%$ of American medical investment is used to cure chronic diseases. With the improvement of living standards, the incidence rate of chronic diseases becomes higher and higher, with the expense on the chronic diseases in the USA each year being 2700 billion dollars, which accounts for $18 \%$ of the annual GDP of the USA.

In fact, doctors and medical facilities only solve $10 \%$ of medical problems, with another $50 \%$ depending on living habits, daily schedules and exercise and the other $20 \%$ depending on heredity. As for personal living habits, doctors can only give some advice, but we can solve this problem by using the big data to guide preferentially, and in fact, most of the medical problems can be solved through the big data.

\subsection{Correct Data}

The medical field is full of opportunities, so we need to collect correct data to solve this problem.

First, the data is great and the data source is wide, including the government, insurance companies, finance, data from the hospitals (including electronic medical records, medical images, etc.), and even personal social media fields. The medical field has stricter requirements on the quality and accuracy of the data than other fields.

However, the medical field is full of much dark data, because human-related data is really sensitive (individuals or hospitals are unwilling to share them, and others are unable to see them behind the scenes), mainly including securityrelated and privacy-related problems. In order to get such data, the protection of personal privacy, namely the security problem, is also one aspect of the problem. 


\subsection{Correct Technology and Methods}

Our health surveillance model is established on the basis of the big data analysis, with the raw data first being cleaned and gathered and then selected and transformed, and the disease prediction is made with the method of data mining. For example, the physical examination data in recent years is able to find out the high risk groups and realize the visualization and evaluation of the data.

\subsection{Correct Decisions}

Traditionally, we always try to find out the reasons only when something is happened or after something has happened. We hope that we can make predictions before the happening of the events, that is to say, to find out the reasons and sources of the events that have happened or are happening and predict the possibility of the events that havent happened. Our ultimate purpose is to make predictions before the happening of events. As for precision medicine, we hope to wipe out the diseases in advance, and that is one of the important values of the big data analysis.

\section{Samrt Clothing}

The key to the emotion communication is to collect the signals of the human bodies, and the key to the collection of physiological signals of the human bodies is the comfortableness. For example, if we ask you to wear some sensors, you will feel really uncomfortable, so we need to be able to use some new technology to solve this problem.

The previous wearable devices are uncomfortable, inconvenient for users to carry and operate, and of single function, so users need to take more part in the operation and maintenance of the devices. The Wearables 2.0 we put forward uses Smart Clothing as the carrier and integrates various kinds of sensors into the clothes to make the users not feel the existing of the wearable devices, realizing the collection of the data unconsciously. We can use Wearables 2.0 to collect ECG Signals, and upload the physiological signals of the wearers to the intelligent devices or the cloud in real time, providing mobile health or sport management monitoring anytime anywhere in their daily life.

Our ARM-based low power consumption microprocessors have designed ECG collecting devices, mainly including two components: one is the amplifying circuit (since ECG signals are really weak, we need to use the amplifying circuit to amplify the ECG signals), and the other is ADC sampling circuit.

It offers flexible ECG collecting plans on the basis of the Smart Clothing:

- At ordinary times, it uses two textile dry electrodes on the Smart Clothing to collect ECG signals. This kind of collecting method is suitable for the longterm ECG signal monitoring. 
- When the emergency happens, we need to increase the precision of the collected ECG signals, so at this time, the users can access the third electrode. The third electrode is an ordinary electrode, with it being able to be removed at any time after its used.

The framework and communication process of the ECG signal collection:

- First, we use ECG collecting devices to collect users original ECG signals and digitalize them. Then we can choose to transfer through serial ports or bluetooth.

- If we transfer through serial ports, we need to transfer the signals to the PC, with the PC being able to decide whether to upload the real-time ECG to the cloud at the same time of drawing ECG waveform by the display program.

- If we transfer through Bluetooth, we need to transfer the signals to the smart phones, with the smart phones uploading the ECG data to the cloud for storage at the same time of drawing ECG waveform by the phone program.

- On the cloud, the original ECG originals will be stored in the database (its convenient for further analysis), and meanwhile, there will be a visual WEB interface to show the uploaded heart rate in real time.

In general, the Smart Clothing is comfortable, washable, reliable, durable, affordable, fashionable, etc., and it's crucial for the emotion care, emotion games and big data care. We are able to integrate humans with the cloud seamlessly through the Smart Clothing.

\section{Emotion-Care Robots}

Just like IoT, the emotion-care robots include three parts: the sensing layer (sensing physiological signals, living habits, etc.), the network layer (transferring information), and the cloud (analyzing the data); however, we add an actionfeedback layer to this, namely Emotion Feedback, with which the robots will be able to adjust your moods and states according to your present emotions to care your emotions. So the Emotion Feedback is the key point of the whole robot system.

The design concept of the robots: on the basis of the existing health surveillance system, the front end adopts low-cost robots, while the back end introduces powerful cloud computing technology to provide the users with efficient, flexible, accurate and easy-to-use health surveillance service. These robots meet the needs of two kinds of people:

- Empty-nest elderly. The aged is able to communicate with the robots, talk with their children far away, and even surf the Internet and watch TV.

- Children far away. They can have a remote control on the robots. For example, if their parents are in the toilet without mobile phones, and they are really worried because they are unable to get in touch with their parents, then the loudspeakers on the robots will call them first, turn on the cameras if there 
is no response, and theyre able to control the movement of the robots to look for their parents.

Our purpose is to let the robots take the place of the simple-functional communication terminals, and with the help of various kinds of sensors, the robots will collect and store the parameters such as physiological status, eating habits, exercise conditions, and family environment in real time, and upload them to the monitoring center, with the cloud computing technology realizing the storage and calculating of the old peoples health data and the comprehensive monitoring of their health. Up to now, we have designed three generations of robots, from the four-wheel robots that can have voice interactions, to four-wheel robots that have the functions of information collection, voice interaction and video monitoring, to biped walking robots that can have voice interactions. We have established a 4G-based test platform on the basis of the cloud big data all-in-one machine. The robots can be connected to the data center through a $4 \mathrm{G}$ mobile phone and a LTE base station, and the final control instructions will be reported back to the robots through the data center, and meanwhile, they will make use of the available resources around to comfort peoples emotions. At the same time, it realizes the emotion interaction based on the wearable computing and cloud computing technology (on the platform, the 4G LTE communication modules on the robots will be connected with the data center through the LTE base station, and the cloud will guide the robots to give feedbacks. Next, we are going to increase the wisdom of the robots and improve the accuracy of indentifying emotions, which need plenty of Machine Learning Algorithm to realize.

\section{Emotion Detection Networking}

The emotion detection networking uses the heterogeneous networks and computing resources to collect the emotion big data, analyze the emotions and give emotion feedbacks. These heterogeneous networks and computing resources can be divided into mobile devices, ad hoc cloudlet, local cloud and remote cloud according to their hierarchical relationships.

The tasks involved in the emotion communication include three different kinds:

- The collection of emotion data: it decides where to collect the personal emotion data, with such data being able to come from wearable devices, social data from the social networks, and multimedia information that implies humans emotions such as facial videos.

- Emotion analysis: it uses various kinds of machine learning methods to indentify and analyze the emotions.

- Emotion feedbacks: according to the results of the emotion analysis, it uses a series of hardware resources around the users to activate the emotion feedback actions. 
Then which device should be used to transfer and calculate the data involved in the emotion communication? First, we can divide the complicated task into several subtasks, with them being able to be done in the mobile device, ad hoc cloudlet, local cloud or remote cloud. Traditionally, most of the subtasks depend on the cloud platform, but neglect the communication overhead caused by transferring plenty of data to the cloud, and at the same time, the delay caused by the communication makes users experience degree lower. Since the mobile device, local cloud and remote cloud have advantages and disadvantages respectively, we can decide to assign subtasks to the mobile device, local cloud and remote cloud according to different network environment and users demands; considering many aspects such as the network conditions, communication delay, energy consumption and users experience degrees, to select an optimized task assignment plan is a challenging problem.

\section{References}

[1] M. Chen, Y. Ma, Y. Hao, Y. Li, D. Wu, Y. Zhang, and E. Song, "Cprobot: Cloud-assisted pillow robot for emotion sensing and interaction," in International Conference on Industrial IoT Technologies and Applications. Springer, 2016, pp. 81-93.

[2] M. Chen, P. Zhou, and G. Fortino, "Emotion communication system," IEEE Access, vol. 5, pp. 326-337, 2017.

[3] M. Chen, Y. Zhang, Y. Li, S. Mao, and V. C. Leung, "Emc: Emotion-aware mobile cloud computing in 5g," IEEE Network, vol. 29, no. 2, pp. 32-38, 2015.

[4] Y. Peng, L. Peng, P. Zhou, J. Yang, S. M. M. Rahman, and A. Almogren, "Exploiting energy efficient emotion-aware mobile computing," Mobile Networks and Applications, pp. 1-12, 2017.

[5] M. Chen, Y. Ma, J. Song, C.-F. Lai, and B. Hu, "Smart clothing: Connecting human with clouds and big data for sustainable health monitoring," Mobile Networks and Applications, vol. 21, no. 5, pp. 825-845, 2016.

[6] Y. Miao, W. Li, D. Tian, M. S. Hossain, and M. F. Alhamid, "Narrow band internet of things: Simulation and modelling," IEEE Internet of Things Journal, 2017.

[7] Y. Ma, Y. Wang, J. Yang, Y. Miao, and W. Li, "Big health application system based on health internet of things and big data," IEEE Access, vol. 5, pp. 7885-7897, 2017.

[8] M. Chen, J. Yang, Y. Hao, S. Mao, and K. Hwang, "A 5g cognitive system for healthcare," Big Data and Cognitive Computing, vol. 1, no. 1, p. 2, 2017.

[9] J. L. Y. M. M. H. M. A. Jun Yang, M. Liu, "Botanical internet of things: Towards smart indoor farming by connecting people, plant, data and clouds," Mobile Networks and Applications, 2017.

[10] J. Hogland and N. Anderson, "Function modeling improves the efficiency of spatial modeling using big data from remote sensing," Big Data and Cognitive Computing, vol. 1, no. 1, p. 3, 2017. 
[11] M. Chen, J. Wang, K. Lin, D. Wu, J. Wan, L. Peng, and C. Youn, "Multipath planning based transmissions for iot multimedia sensing," in Proc. IEEE IWCMC, 2016, pp. 1-10.

[12] M. Liu, K. Lin, J. Yang, D. Xiao, Y. Miao, L. Wang, W. Li, Z. Wei, and J. Lu, "Mobile cloud platform: Architecture, deployment and big data applications," Proc. Cloudcomp, 2016. 